\title{
Lymphangiogenesis, Lymphatic Endothelial Cells and Lymphatic Metastasis in Head and Neck Cancer - A Review of Mechanisms
}

\author{
Zhuang Zhang ${ }^{1,2}$, Joseph I. Helman ${ }^{3}$, Long-jiang $\mathrm{Li}^{1 *}$ \\ ${ }^{1}$ State Key Laboratory of Oral Diseases, West China Hospital of Stomatology, Sichuan University, Chengdu, China \\ ${ }^{2}$ Department of Oral and Maxillofacial Surgery, the Medical School Hospital of Qingdao University, Qingdao, China \\ ${ }^{3}$ Section of Oral and Maxillofacial Surgery, University of Michigan, Ann Arbor, USA
}

\begin{abstract}
Lymphatic metastasis is a continuous and complicated process. The detailed mechanisms of lymphatic metastasis are still not very clear, despite considerable research efforts in recent years. Previously, it was commonly accepted that there were no lymphatic vessels in the primary tumor. However, recent studies have demonstrated that lymphatic vessels are detectable in certain types of cancer, and more and more evidence has shown that cancer cells invade into
\end{abstract}

local lymph nodes mainly via peritumoral lymphatic vessels. Moreover, activated endothelial cells may also be important, having an influence on lymphatic metastasis of cancer cells. This article, based on recent research findings, provides an in-depth discussion of the relationship between lymphangiogenesis, tumor-derived lymphatic endothelial cells and lymphatic metastasis in head and neck cancer.

Keywords lymphangiogenesis, lymphatic endothelial cell, lymphatic metastasis, head and neck cancer

Received Oct. 15, 2009; Revision accepted Jan. 11, 2009

\section{Introduction}

The lymphatic system plays multiple roles, including a role in tissue homeostasis, supplemental circulation, and immune surveillance ( $\mathrm{Ji}, 2008)$. Lymphatic vessels, however, may also act as conduits for cancer cells to escape from the primary tumors in a number of carcinomas (Achen and Stacker, 2008). For head and neck squamous cell carcinoma (HNSCC), lymphatic spread is more important than other routes because malignant cells preferentially metastasize to roughly 400 lymph nodes in the cervical region. Lymph node spread is the strongest prognostic factor for survival of patients with HNSCC. Until now, the details of the processes and molecular mechanisms of lymphatic metastasis have been little understood. Several reports on HNSCC showed that lymphangiogenesis was closely related to lymphatic metastasis, lymphatics providing additional conduits for dissemination of cancer cells (Miya- hara et al., 2007; O'Donnell et al., 2008; Zhao et al., 2008; Frech et al., 2009). Moreover, altered phenotypes of lymphatic endothelial cells (LECs) in HNSCC contribute to the lymphatic dissemination of cancer cells. This article focuses on the lymphangiogenesis and specific phenotypes of LECs in HNSCC, with a detailed discussion of their function in the lymphatic dissemination of cancer cells.

\section{Lymphangiogenesis in HNSCC}

Due to the relative lack of efficient and objective methods, research on lymphatic vessels lags far behind that on blood vessels. Previously, many researchers believed that a tumor could not induce lymphangiogenesis because there were no lymphatic vessels in tumors. In the last 15 years, with the application of specific antibodies against LECs, the concept of lymphangiogenesis in tumors has 
been promoted and gradually accepted by many. Lymphangiogenesis is the growth of newly formed lymphatic vessels, a process with multiple steps similar to that of angiogenesis: endothelial cell migration, proliferation and rearrangement, along with degradation, reconstruction and production of extracellular matrix. The growth of lymphatic vessels has been observed in various normal and pathologic processes, such as wound healing, inflammation and tumor progression (Wong et al., 2005; Maruyama et al., 2007).

To investigate whether intratumoral lymphatic vessels existed in xenotransplanted tumor, MDA435 and MCF-7 breast carcinoma cells with overexpression of vascular endothelial growth factor-C (VEGF-C) were orthotopically transplanted in nude mice (Karpanen et al., 2001; Mattila et al., 2002). The results showed that transplanted cancer cells induced the formation of lymphatic vessels in tumors. The dose and intensity of lymphagiogenic factors, however, were not equal with those in naturally occurring breast carcinomas. Therefore, many researchers have focused on the study of lymphagiogenesis in human tumors. At present, immunohistochemistry studies of lymphangiogenesis have been performed in many types of cancers, including breast cancer, pancreatic endocrine tumors, renal cell cancer, liver cancer, prostate cancer and so on (table 1), but the conclusions have been controversial, because lymphangiogenesis cannot always be detected in every malignant tumors of epithelial origin. In other words, it does not always occur, even for the same kind of cancer. Although different markers used in different studies and the poorly differentiated lymphatic morphology might result in these discrepancies, genuine differences in the biology of various cancers must be considered.

For HNSCC, lymphangiogenesis have been detected in most clinical samples. Several reports showed that both intratumoral and peritumoral lymphatic vessels were identified in tumor samples, and were heterogeneously distributed within tumors (Audet et al., 2005; Xuan et al., 2005; Miyahara et al., 2007; O'Donnell et al., 2008; Zhao et al., 2008). In the peritumoral regions, large open lymphatic vessels were frequently identified. Intratumoral lymphatics, however, were either within sheets of tumor cells in carcinomas with a pushing margin and in areas containing leukocyte infiltration in carcinomas with an invasive margin. Additionally, peritumoral lymphatics were found to have more dilated, open lumina than intratumoral lymphatics. In contrast, intratumoral lymphatics had numerous tiny ill-defined lumina, often composed of two to three endothelial cells. None of the peritumoral lymphatics contained proliferating nuclei, while intratumoral lymphatics were proliferative.

\section{Lymphangiogenic factors involved in lym- phangiogenesis}

Similar to tumor-induced angiogenesis, proliferation of the lymphatics is an active biological behavior of tumor cells, with a heterogeneity of interactions of tumor cells with blood vessels and lymphatic vessels in tumors. To date, the exact initiating mechanism of proliferation has not been established. Many studies, however, revealed that cancer cells could release lymphangiogenic growth factors, mainly including VEGF-C, D and A. In HNSCC, VEGF-A, C and D positive cells ranged from being present in very small numbers to being present throughout almost the entire tumor, and VEGF-C and D expression were frequently upregulated at the invasive tumor front (Shintani et al., 2004). These lymphangiogenic growth factors were able stimulate the development of lymphatic vessels by different pathways (Nakaya et al., 2005). For example, VEGF-A controls endothelial cell behaviors by binding with vascular endothelial growth factor receptor-1 (VEGFR-1) and VEGFR-2, affecting proliferation, migration, specialization and survival; VEGF-C and VEGF-D bind to and activate both VEGFR-3 and VEGFR-2, but not VEGFR-1 (Li and Eriksson, 2001). In addition, VEGF-C and VEGF-D bind to VEGFR-2 with a lower affinity than they bind to VEGFR-3 (Witmer et al., 2003).

VEGF-C is a primarily lymphangiogenic factor, inducing the growth of lymphatic vessels in normal and pathologic conditions. In xenotransplanted tumors, cancer cells transfected by VEGF-C gene induce the growth of functional lymphatics and result in hyperplastic vessels, indicating that VEGF-C is a potent inducer of tumor lymphangiogenesis (Cohen-Kaplan et al., 
Table 1 The clinical studies of lymphangiogenesis in malignant tumors

\begin{tabular}{|c|c|c|c|}
\hline Tumor type & Markers & Results & Author and Reference \\
\hline Breast cancer & D2-40/Ki-67 & $\mathrm{Y}$ & van der Auwera et al., 2004 \\
\hline Breast aancer & $\mathrm{D} 2-40$ & Y & Britto et al., 2009 \\
\hline Breast cancer & LYVE-1/CD34/Ki-67 & $\mathrm{N}$ & Williams et al., 2003 \\
\hline Breast cancer & LYVE1 & Y & Bono et al., 2004 \\
\hline Breast cancer & Podoplanin & $\mathrm{N}$ & Schoppmann et al., 2001 \\
\hline Breast cancer & PCNA/D2-40 & $\mathrm{N}$ & Agarwal et al., 2005 \\
\hline Pancreatic cancer & Ki-67/LYVE-1 & $\mathrm{Y}$ & Sipos et al., 2005 \\
\hline Renal cell cancer & VEGFR-3 & $\mathrm{Y}$ & Bando et al., 2004 \\
\hline Prostate cancer & LYVE-1/CD34 & $\mathrm{N}$ & Trojan et al., 2004 \\
\hline Liver cancer & $\mathrm{D} 2-40$ & Y & Thelen et al., 2009 \\
\hline Lung cancer & LYVE-1 and the MIB1 & $\mathrm{N}$ & Koukourakis et al., 2005 \\
\hline Colorectal cancer & 5'-Nase-ALPase & $\mathrm{Y}$ & Jia et al., 2004 \\
\hline Esophageal cancer & Podoplanin & Y & Nakayama et al., 2007 \\
\hline HNSCC & LYVE-1 & Y & Frech et al., 2009 \\
\hline HNSCC & Podoplanin/CD34/Ki-67 & Y & Kyzas et al., 2005 \\
\hline HNSCC & LYVE-1 & $\mathrm{Y}$ & Audet et al., 2005 \\
\hline HNSCC & D2-40 & Y & Franchi et al., 2004 \\
\hline Oral cancer & PA2.26 & Y & Munoz-Guerra et al., 2004 \\
\hline Oral cancer & D2-40 & Y & Xuan et al., 2005 \\
\hline Oral cancer & $\mathrm{D} 2-40$ & Y & Miyahara et al., 2007 \\
\hline Oral cancer & $\mathrm{D} 2-40$ & $\mathrm{Y}$ & Zhao et al., 2008 \\
\hline Oral cancer & Podoplanin/CD34 & Y & O'Donnell et al., 2008 \\
\hline Cutaneous melanoma & LYVE-1/Ki-67 & $\mathrm{Y}$ & Straume et al., 2003 \\
\hline
\end{tabular}

* PCNA proliferating cell nuclear antigen.

2008). Clinical evidence also suggests that tumor lymphangiogenesis is associated with the expression of VEGF-C in cancer cells, including breast cancer (Nakamura et al., 2005), oral cancer (Sugiura et al., 2009), non-small-cell lung cancer (Lu et al., 2005), pancreatic adenocarcinoma (Zhang et al., 2007), and colorectal cancer (Fukunaga et al., 2006). Several studies, however, have contradicted the above observations. In primary uveal melanomas, although VEGF-C and its receptor VEGFR-3 were expressed, neither lymphatics nor signs of lymphangiogenesis were evident, suggesting that the concerted action of these players was not sufficient for lymphangiogenesis to occur in this type of tumor (Clarijs $e t$ al., 2001). In a model of mouse tail skin regeneration, excessive VEGF-C expression did not enhance the rate of LEC migration and the density of lymphatic vessels (Goldman et al., 2005). Furthermore, in the murine dorsal skinfold chamber, B16F10 melanomas cell secreted excess VEGF-C, but genuine functional lymphatic vessels did not exist, which displayed a retrograde draining pattern (Isaka et al., 2004). How then does one explain the different results and understand the biologic function of VEGF-C? It has been hypothesized that the different vectors used in animal experiments might contribute to the heterogeneity of results, because the target tissues, in which the vector is optimally expressed, were rather different. For example, instead of adenoviral vectors, AAV infection could give long-term transgenic expression without cell-mediated immune response or toxicity. Additionally, proteolytic processing is a 
regulator of VEGF-C activity and a mature form enhanced the binding and cross-linking of VEGFR-2 and VEGFR-3 in comparison to full length material. Because of distinct proteolytic processing in different tissues, biological effects of VEGF-C might not be the same.

Like VEGF-C, the affinity of VEGF-D toward its receptors is also regulated by proteolytic processing. Induction of VEGF-D is mainly mediated, however, by direct cell-cell contact. The capacity of VEGF-D to promote lymphangiogenesis is tissue-specific and dependent on the abundance of blood vessels, and receptor expression of lymphatics for VEGF-D in a given tissue (Rissanen et al., 2003). Functional autocrine stimulation of VEGF-D in cancer not only stimulates the proliferation of cancer cells and LECs, but also plays a role in the maintenance of antiapoptotic characteristics of tumor-derived endothelial cells. An animal model has provided direct experimental evidence that increased levels of VEGF-D promote active tumor lymphangiogenesis and lymphatic metastasis (Achen and Stacker, 2008). By blocking VEGFR-3 signaling, lymphangiogenic effect can be suppressed (He et al., 2005). Immunohistochemical studies in kidney cancer and gastric cancer have shown that VEGF-D was mainly expressed in cancer cells and in VEGFR3positive vessels adjacent to immunopositive tumor cells, but not in vessels distant from the tumors, which suggests that VEGF-D plays a role in the regulation of lymphangiogenesis (Bierer et al., 2008; Choi et al., 2008).

Traditionally, VEGF-A is believed to mainly initiate the process of vascularization by stimulating chemoattraction and proliferation of angioblasts and endothelial cells (Nakazato et al., 2006). Whether it has an effect on lymphatic endothelial cell proliferation has been a controversial matter. Some studies have shown that VEGF-A stimulated formation of disorganized, nascent vasculatures with only a few lymphatic vessels (Cao et al., 2004). Other studies have shown that transgenic expression of VEGF-A induced proliferation and persistent enlargement of lymphatic vessels, which closely resembles the lymphatic phenotype in human psoriatic skin (Kunstfeld et al., 2004). Recently, VEGF-A has also been confirmed to exert potent lymphangiogenic activity by activating
VEGFR-2, thereby facilitating metastatic spread (Hirakawa et al., 2005). Several studies revealed that VEGFR-2 was expressed on cultured LECs and in cutaneous lymphatic vessels and VEGF-A stimulated LECs proliferation (Petrova et al., 2002; Hirakawa et al., 2003). In the VEGF-A transgenic mice, blocking antibodies against VEGFR-1 and VEGFR-2 potently inhibited lymphatic vessel enlargement. In addition, VEGF-A enhanced the heterodimerization of VEGFR-3 with VEGFR-2 and the phosphorylation of VEGFR-3, therefore providing proliferative stimuli to the LECs (Alam et al., 2004).

\section{Lymphangiogenesis and lymphatic meta- stasis in HNSCC}

Cancer cells in the primary tumor have a long way to go before they obtain the ability to metastasize and successfully disseminate. In general, the anatomic pathway of lymphatic dissemination is as follows. With tumor progression, cancer cells secrete lymphangiogenic cytokines which result in the formation of lymphatic vessels around or within the tumor. Then, cancer cells dissociate from the primary tumor and invade the extracellular matrix. Following the chemotactic gradient of chemokines in tissue, cancer cells move toward lymphatic vessels. After attaching to lymphatic endothelium, they cross the endothelia cell barrier and enter into the lymphatic lumen. Then the cancer cells in lymphatic vessels, singly or in clusters, are drained into sentinel lymph nodes within the lymphatic stream. During this process, the phenotypes of cancer cells consistently alter. For instance, they become more autonomous and are resistant to hypoxic environments, they secrete the proteolytic enzymes for local invasion, express specific adhesion molecules, produce lympangiogenic factors to attain a transport pathway and eventually evade the host defense. Therefore, lymphatic metastasis is a continuous and complicated process.

For most carcinomas, transport of cancer cells via lymphatic vessels is the most common pathway, following routes of natural drainage, because the lymphatic system seems to have more advantages over blood circulation for cancer dissemination. Unlike blood capillaries, initial lymphatics are much 
larger and lack a continuous basal membrane. Additionally, tumor cells in the lymphatic vessels are not prone to serum toxicity, high shear stress, or mechanical deformation. Lymphatic spread of HNSCC is more important, however, than in other tumors because they preferentially metastasize to roughly 400 lymph nodes in this area.

During the progression of HNSCC, lymph vessels are repeatedly destroyed and regenerated with the invasion of cancer cells (Nakaya et al., 2005). Thus, key questions must be answered: What is the function of lymphangiogenesis in the lymphatic metastasis of HNSCC? Does the lymphangiogenesis contribute to lymphatic dissemination by providing additional channels? Previous studies have shown that lymphangiogenesis was closely associated with an increased risk of lymph node metastasis. If lymphangiogenesis-related properties of LECs were inhibited, the risk of lymphatic dissemination was significantly reduced (Wen et $a l$. , 2009). Therefore, cancer-induced lymphangiogenesis is essential for the invasion and secondary lymphatic metastasis. It is unclear, however, whether this is a consequence of intratumoral lymphatics, peritumoral lymphatics, or both.

In HNSCC, lymphatic vessels have been found to be more numerous and larger in the peritumoral area than within the tumor itself. The number and relative area of intratumoral and peritumoral lymphatics have been found to be significantly higher in HNSCC cases with lymph node metastasis (Franchi et al., 2004). Statistic analysis has confirmed that high peritumoral lymphangiogenesis is associated with an increased risk of developing lymph node metastasis, suggesting that peritumoral lymphatics are major drainage channels for cancer cells. But some studies have shown that there is a significant relationship between the presence of intratumoral lymphatics and nodal metastases in patients with laryngeal carcinoma (Audet et al., 2005), and that patients with intratumoral lymphatic-positive tumors had a less favorable disease-free pattern compared with patients with intratumoral lymphatics-negative tumors (Munoz-Guerra et al., 2004). These results suggest that intratumoral lymphatics played a greater role than peritumoral lymphatics in nodal metastasis of HNSCC (Maula et al., 2003). Additionally, others have argued that the spread of
HNSCC cells to lymph nodes might involve invasion of both peritumoral and intratumoral vessels, because they believed that it was possible that some of the emboli observed in peritumoral vessels originated from initial invasion of intratumoral vessels, although tumor emboli were occasionally observed within peritumoral vessels and not obvious within intratumoral vessels.

It is evident from the structural and morphological characteristics of peritumoral vessels that they are more easily invaded. Lymphatic metastasis requires, however, a functional lymphatic network, and the condition of lymphatic drainage is therefore a relevant factor for lymphatic metastasis (Maza et al., 2003; He et al., 2004). In B16F10 melanomas murine model, hyperplastic peritumoral lymphatics were shown to be functional, although lymphatic vessels displayed a retrograde draining pattern (Isaka et al., 2004). Other studies also confirmed that the lack of functional lymphatics in tumors was a common phenomenon, while functional lymphatics were found to exist in the tumor margin (Padera et al., 2002). More importantly, even when there are no functional lymphatics in a tumor, lymphatic metastasis can still occur. Therefore, we believe that cancer cells may spread via peritumor lymphatics, and intratumoral lymphatics should be regarded as an additional pathway, rather than a necessity, for metastasis (Achen et al., 2005). At present, the mechanism of intratumoral lymphatics dysfunction is not entirely known. It is hypothesized that the rapid growth of tumor results in tissue edema, which generates mechanical forces to compress the lymphatic. Additionally, tumor cells might destroy the lymphatic structure, and the newly formed valves of intratumoral lymphatic are then incomplete and nonfunctional.

\section{Lymphatic endothelial cells might play a positive role in lymphatic metastasis}

Although lymphatic vessels constitute the most important channel of lymphatic spread, lymphatic endothelium is an interactive surface for cancer cells, and the ability of cancer cells to interact with the LEC is a key step in allowing them to invade the lymphatic system. In 1990, Hartveit observed that tumor cells were washed with the tide of 
tissue fluid into the lymphatic drainage channels. Moreover, interstitial fluid pressure (IFP) in solid tumors was significantly elevated compared to normal tissues and increased as tumors increased in size, which facilitated tumor cell intravasation and promote metastasis (Lunt et al., 2008; Ferretti et al., 2009). These results suggest that lymphatic invasion is not an active process, but is closely associated with the functional status of LECs. For example, enhanced IFP results in increase of interstitial fluid volume (IFV). Thus, the anchoring filaments are stretched and junctions of the endothelial cells opened, allowing cancer cells to enter into the lymphatic vessels. Our studies have shown that open junction was the main junction type in peritumoral lymphatics (about 42\%), which had a greatly enlarged opening space of $0.3-5 \mu \mathrm{m}$. The overlapping junction became the second most common junction type in peritumor tissues $(38 \%)$. The proportion of inlaid junctions was $12 \%$, and was $8 \%$ for end-end junctions. Therefore, lymphatic endothelium itself might have an important influence on the lymphatic metastasis of cancer cells.

With the development of molecular biology, researchers have come to gradually understand the functions of LECs in lymphatic metastasis. Recent studies have shown that integrin $\alpha 9 \beta 1$ is expressed on LEC induced cancer cell migration by binding with plasmin (Majumdar et al., 2004), and promotes lymphangiogenesis and lymphatic metastasis by binding with VEGF-C and D (Vlahakis et al., 2005). The mannose receptor (MR), lymphatic vessel endothelial hyaluronan receptor (LYVE)-1 and common lymphatic endothelial and vascular endothelial receptors (CLEVER)-1 have roles beyond the lymphatic system, directing the traffic of cancer cells into lymphatics (Jackson et al., 2001; Irjala et al., 2003; Guo et al., 2005). LECs constitutively secrete different cytokines of the CXC, CC and C subfamilies, such as secondary lymphoid tissue chemokine (SLC)/CCL21, macrophage inflammatory protein (MIP)-3/CCL20 (Zhuang et al., 2009). These data suggest that LECs are responsible for the invasion and lymphatic metastasis of cancer cells.

Recent studies have shown that LECs in HNSCC have a remarkable degree of phenotypic plasticity, characterized by elevated expression of endothelial specific adhesion molecules, the transforming growth factor-beta coreceptor Endoglin (CD105) and the angiogenesis-associated leptin receptor (Clasper et al., 2008). Our previous study also showed that in contrast to LEC, oral tongue cancer-induced LECs were more proliferative and had enhanced ability of organizing capillary-like structures (Zhuang et al., 2008). Moreover, LEC phenotypes changed with the enhancement of metastatic potential accordingly. These data suggested that LECs in tumor are distinct from normal LECs and have a specific phenotype. Therefore, it is hypothesized that the phenotypes of LEC could be induced by cancer cells to encourage the lymphatic dissemination of cancer cells.

HNSCC have an affinity for lymphatic vessels, and it is questioned whether LECs in tumors play an important role in guiding cancer cells migration to lymphatic vessels. It is well known that cancer metastasis is not a random process, and chemotaxis is an essential component of cancer cell trafficking and metastasis. It is assumed that cancer cells actively crawl towards blood and lymphatic vessels following the attractant molecule gradients formed by endothelial cells (Condeelis and Pollard, 2006). More and more evidence suggests that directed movement caused by chemokines is required for the formation of tumor metastasis. For example, CCL2 regulates invasion and migration of cancer cells by binding to chemokine receptors CCR4 (Ishida and Ueda, 2006; Loberg et al., 2007); CCL20/CCR6 ligand-receptors are involved in liver metastasis of colorectal cancer (Rubie $e t$ al., 2006); CXCL2 provokes a dose-dependent increase of cell migration and a most pronounced cell adhesion in vitro (Kollmar et al., 2006); high expression of CXCL5 in gastric cancer results in lymph node metastasis (Park et al., 2007); CXCL6 has an important role in the growth and metastasis of small cell lung cancer (Zhu et al., 2006). Recently, we found that CXCL1, CXCL5, CXCL6, CCL2, CCL7, CCL17 and CCL20 were upregulated at mRNA or protein level in tongue cancer cell induced LECs (Zhuang et al., 2009), indicating that LECs in tumor could secreted chemokines to facilitate the directed migration of tongue cancer cells, helping to explain why cancer cells have a predilection for lymphatic metastasis. 


\section{Conclusion}

To date, the mechanism of lymphatic metastasis is still unclear, but we believe that the changes of lymphatic vessels and LECs induced by tumors are key factors in this process. Both peritumoral and intratumoral lymphatics, however, provide at most a gateway, and do not decisively influence successful lymphatic metastasis because successful dissemination of cancer cells mainly depends on the interaction between cancer cells and LECs. Therefore, future research should focus on the altered phenotypes of LECs induced by tumor cells and uncover the key factors related to lymphatic metastasis.

\section{References}

Achen MG, Stacker SA (2008). Molecular control of lymphatic metastasis. Ann N Y Acad Sci, 1131: 225234.

Achen MG., McColl BK, Stacker SA (2005). Focus on lymphangiogenesis in tumor metastasis. Cancer Cell, 7(2): 121-127.

Agarwal B, Saxena R, Morimiya A, Mehrotra S, Badve S (2005). Lymphangiogenesis does not occur in breast cancer. Am J Surg Pathol, 29(11): 1449-1455.

Alam A, Herault JP, Barron P, Favier B, Fons P, DelesqueTouchard N, et al. (2004). Heterodimerization with vascular endothelial growth factor receptor-2 (VEGFR-2) is necessary for VEGFR-3 activity. Biochem Biophys Res Commun, 324(2): 909-915.

Audet N, Beasley NJ, MacMillan C, Jackson DG, Gullane PJ, Kamel-Reid S (2005). Lymphatic vessel density, nodal metastases, and prognosis in patients with head and neck cancer. Arch Otolaryngol Head Neck Surg, 131(12): 1065-1070.

Bando H, Brokelmann M, Toi M, Alitalo K, Sleeman JP, Sipos B, et al. (2004). Immunodetection and quantification of vascular endothelial growth factor receptor-3 in human malignant tumor tissues. Int $J$ Cancer, 111(2): 184-191.

Bierer S, Herrmann E, Kopke T, Neumann J, Eltze E, Hertle L, et al. (2008). Lymphangiogenesis in kidney cancer: expression of VEGF-C, VEGF-D and VEGFR-3 in clear cell and papillary renal cell carcinoma. Oncol Rep, 20(4): 721-725.

Bono P, Wasenius VM, Heikkila P, Lundin J, Jackson DG,
Joensuu H. (2004). High LYVE-1-positive lymphatic vessel numbers are associated with poor outcome in breast cancer. Clin Cancer Res, 10(21): 7144-7149.

Britto AV, Schenka AA, Moraes-Schenka NG, Alvarenga M, Shinzato JY, Vassallo J, et al. (2009). Immunostaining with D2-40 improves evaluation of lymphovascular invasion, but may not predict sentinel lymph node status in early breast cancer. BMC Cancer, 9, 109.

Cao R, Eriksson A, Kubo H, Alitalo K, Cao Y, Thyberg J (2004). Comparative evaluation of FGF-2-, VEGF-A-, and VEGF-C-induced angiogenesis, lymphangiogenesis, vascular fenestrations, and permeability. Circ Res, 94(5): 664-670.

Choi JH, Oh YH, Park YW, Baik HK, Lee YY, Kim IS (2008). Correlation of vascular endothelial growth factor-D expression and VEGFR-3-positive vessel density with lymph node metastasis in gastric carcinoma. J Korean Med Sci, 23(4): 592-597.

Clarijs R, Schalkwijk L, Ruiter DJ, de Waal RM (2001). Lack of lymphangiogenesis despite coexpression of VEGF-C and its receptor Flt-4 in uveal melanoma. Invest Ophthalmol Vis Sci, 42(7): 1422-1428.

Clasper S, Royston D, Baban D, Cao Y, Ewers S, Butz S, et al. (2008). A novel gene expression profile in lymphatics associated with tumor growth and nodal metastasis. Cancer Res, 68(18): 7293-7303.

Cohen-Kaplan V, Naroditsky I, Zetser A, Ilan N, Vlodavsky I, Doweck I (2008). Heparanase induces VEGF $\mathrm{C}$ and facilitates tumor lymphangiogenesis. Int J Cancer, 123(11): 2566-2573.

Condeelis J, Pollard JW (2006). Macrophages: obligate partners for tumor cell migration, invasion, and metastasis. Cell, 124(2): 263-266.

Ferretti S, Allegrini PR, Becquet MM, McSheehy PM (2009). Tumor interstitial fluid pressure as an earlyresponse marker for anticancer therapeutics. Neoplasia, 11(9): 874-881.

Franchi A, Gallo O, Massi D, Baroni G, Santucci M (2004). Tumor lymphangiogenesis in head and neck squamous cell carcinoma: a morphometric study with clinical correlations. Cancer, 101(5): 973-978.

Frech S, Hormann K, Riedel F, Gotte K (2009). Lymphatic vessel density in correlation to lymph node metastasis in head and neck squamous cell carcinoma. Anticancer Res, 29(5): 1675-1679.

Fukunaga S, Maeda K, Noda E, Inoue T, Wada K, Hirakawa K (2006). Association between expression of vascular endothelial growth factor $\mathrm{C}$, chemokine receptor CXCR4 and lymph node metastasis in 
colorectal cancer. Oncology, 71(3/4): 204-211.

Goldman J, Le TX, Skobe M, Swartz MA (2005).

Overexpression of VEGF-C causes transient lymphatic hyperplasia but not increased lymphangiogenesis in regenerating skin. Circ Res, 96(11): 1193-1199.

Guo LX, Zou K, Ju JH, Xie H (2005). Hyaluronan promotes tumor lymphangiogenesis and intralymphantic tumor growth in xenografts. Acta Biochim Biophys Sin (Shanghai), 37(9): 601-606.

He Y, Rajantie I, Ilmonen M, Makinen T, Karkkainen MJ, Haiko P, Salven P, et al. (2004). Preexisting lymphatic endothelium but not endothelial progenitor cells are essential for tumor lymphangiogenesis and lymphatic metastasis. Cancer Res, 64(11): 3737-3740.

He Y, Rajantie I, Pajusola K, Jeltsch M, Holopainen T, Yla-Herttuala S, et al. (2005). Vascular endothelial cell growth factor receptor 3-mediated activation of lymphatic endothelium is crucial for tumor cell entry and spread via lymphatic vessels. Cancer Res, 65(11): 4739-4746.

Hirakawa S, Hong YK, Harvey N, Schacht V, Matsuda K, Libermann T, et al. (2003). Identification of vascular lineage-specific genes by transcriptional profiling of isolated blood vascular and lymphatic endothelial cells. Am J Pathol, 162(2): 575-586.

Hirakawa S, Kodama S, Kunstfeld R, Kajiya K, Brown LF, Detmar M (2005). VEGF-A induces tumor and sentinel lymph node lymphangiogenesis and promotes lymphatic metastasis. J Exp Med, 201(7): 1089-1099.

Irjala H, Alanen K, Grénman R, Heikkilä P, Joensuu H, Jalkanen S (2003). Mannose receptor (MR) and common lymphatic endothelial and vascular endothelial receptor (CLEVER)-1 direct the binding of cancer cells to the lymph vessel endothelium. Cancer Res, 63(15): 46714676.

Isaka N, Padera TP, Hagendoorn J, Fukumura D, Jain RK (2004). Peritumor lymphatics induced by vascular endothelial growth factor-C exhibit abnormal function. Cancer Res, 64(13): 4400-4404.

Ishida T, Ueda R (2006). CCR4 as a novel molecular target for immunotherapy of cancer. Cancer Sci, 97(11): $1139-1146$.

Jackson DG, Prevo R, Clasper S, Banerji S (2001). LYVE-1, the lymphatic system and tumor lymphangiogenesis. Trends Immunol, 22(6): 317-321.

Ji RC (2008). Lymphatic endothelial cells, lymphedematous lymphangiogenesis, and molecular control of edema formation. Lymphat Res Biol, 6(3/4): 123137.
Jia YT, Li ZX, He YT, Liang W, Yang HC, Ma HJ (2004). Expression of vascular endothelial growth factor-C and the relationship between lymphangiogenesis and lymphatic metastasis in colorectal cancer. World $J$ Gastroenterol, 10(22): 3261-3263.

Karpanen T, Egeblad M, Karkkainen MJ, Kubo H, Yla-Herttuala S, Jaattela M, Alitalo K (2001). Vascular endothelial growth factor $\mathrm{C}$ promotes tumor lymphangiogenesis and intralymphatic tumor growth. Cancer Res, 61(5): 1786-1790.

Kollmar O, Scheuer C, Menger MD, Schilling MK (2006). Macrophage inflammatory protein- 2 promotes angiogenesis, cell migration, and tumor growth in hepatic metastasis. Ann Surg Oncol, 13(2): 263-275.

Koukourakis MI, Giatromanolaki A, Sivridis E, Simopoulos C, Gatter KC, Harris AL, et al. (2005). LYVE-1 immunohistochemical assessment of lymphangiogenesis in endometrial and lung cancer. J Clin Pathol, 58(2): 202-206.

Kunstfeld R, Hirakawa S, Hong YK, Schacht V, Lange-Asschenfeldt B, Velasco P, et al. (2004). Induction of cutaneous delayed-type hypersensitivity reactions in VEGF-A transgenic mice results in chronic skin inflammation associated with persistent lymphatic hyperplasia. Blood, 104(4): 1048-1057.

Kyzas PA, Geleff S, Batistatou A, Agnantis NJ, Stefanou D (2005). Evidence for lymphangiogenesis and its prognostic implications in head and neck squamous cell carcinoma. J Pathol, 206(2): 170-177.

Li X, Eriksson U (2001). Novel VEGF family members: VEGF-B, VEGF-C and VEGF-D. Int J Biochem Cell Biol, 33(4): 421-426.

Loberg RD, Ying C, Craig M, Yan L, Snyder LA, Pienta $\mathrm{KJ}$ (2007). CCL2 as an important mediator of prostate cancer growth in vivo through the regulation of macrophage infiltration. Neoplasia, 9(7): 556-562.

Lu ZQ, Li HG, Xie DR, Zhang HZ, Shen XM, Zeng YJ, et al. (2005). Expression and clinical significance of vascular endothelial growth factor $\mathrm{C}$ and vascular endothelial growth factor receptor 3 in non-small cell lung carcinoma. Ai Zheng, 24(9): 1132-1135.

Lunt SJ, Kalliomaki TM, Brown A, Yang VX, Milosevic M, Hill RP (2008). Interstitial fluid pressure, vascularity and metastasis in ectopic, orthotopic and spontaneous tumours. BMC Cancer, 8: 2.

Majumdar M, Tarui T, Shi B, Akakura N, Ruf W, Takada Y (2004). Plasmin-induced migration requires signaling through protease-activated receptor 1 and integrin alpha(9)beta(1). J Biol Chem, 279(36): 37528-37534. 
Maruyama K, Asai J, Ii M, Thorne T, Losordo DW, D'Amore PA (2007). Decreased macrophage number and activation lead to reduced lymphatic vessel formation and contribute to impaired diabetic wound healing. Am J Pathol, 170(4): 1178-1191.

Mattila MM, Ruohola JK, Karpanen T, Jackson DG, Alitalo K, Harkonen PL (2002). VEGF-C induced lymphangiogenesis is associated with lymph node metastasis in orthotopic MCF-7 tumors. Int J Cancer, 98(6): 946951.

Maula SM, Luukkaa M, Grenman R, Jackson D, Jalkanen S, Ristamaki R (2003). Intratumoral lymphatics are essential for the metastatic spread and prognosis in squamous cell carcinomas of the head and neck region. Cancer Res, 63(8): 1920-1926.

Maza S, Valencia R, Geworski L, Sandrock D, Zander A, Audring H, et al. (2003). Influence of fast lymphatic drainage on metastatic spread in cutaneous malignant melanoma: a prospective feasibility study. Eur J Nucl Med Mol Imaging, 30(4): 538-544.

Miyahara M, Tanuma J, Sugihara K, Semba I (2007). Tumor lymphangiogenesis correlates with lymph node metastasis and clinicopathologic parameters in oral squamous cell carcinoma. Cancer, 110(6): 1287-1294.

Munoz-Guerra MF, Marazuela EG, Martin-Villar E, Quintanilla M, Gamallo C (2004). Prognostic significance of intratumoral lymphangiogenesis in squamous cell carcinoma of the oral cavity. Cancer, 100(3): 553-560.

Nakamura Y, Yasuoka H, Tsujimoto M, Imabun S, Nakahara M, Nakao K, et al. (2005). Lymph vessel density correlates with nodal status, VEGF-C expression, and prognosis in breast cancer. Breast Cancer Res Treat, 91(2): 125-132.

Nakaya H, Kawashiri S, Tanaka A, Noguchi N, Kato K, Hase T, et al. (2005). Influences of angiogenesis and lymphangiogenesis on cancerous invasion in experimentally induced tongue carcinoma. J Oral Pathol Med, 34(2): 87-92.

Nakayama Y, Matsumoto K, Nagato M, Inoue Y, Katsuki T, Minagawa N, et al. (2007). Significance of lymphangiogenesis as assessed by immunohistochemistry for podoplanin in patients with esophageal carcinoma. Anticancer Res, 27(1B): 619-625.

Nakazato T, Shingaki S, Kitamura N, Saito C, Kuwano R, Tachibana M (2006). Expression level of vascular endothelial growth factor-C and -A in cultured human oral squamous cell carcinoma correlates respectively with lymphatic metastasis and angiogenesis when transplanted into nude mouse oral cavity. Oncol Rep, 15(4): 825-830.

O'Donnell RK, Feldman M, Mick R, Muschel RJ (2008). Immunohistochemical method identifies lymphovascular invasion in a majority of oral squamous cell carcinomas and discriminates between blood and lymphatic vessel invasion. $J$ Histochem Cytochem, 56(9): 803-810.

Padera TP, Kadambi A, di Tomaso E, Carreira CM, Brown EB, Boucher Y, et al. (2002). Lymphatic metastasis in the absence of functional intratumor lymphatics. Science, 296(5574): 1883-1886.

Park JY, Park KH, Bang S, Kim MH, Lee JE, Gang J, et al. (2007). CXCL5 overexpression is associated with late stage gastric cancer. J Cancer Res Clin Oncol, 133(11): 835-840.

Petrova TV, Makinen T, Makela TP, Saarela J, Virtanen I, Ferrell RE, et al. (2002). Lymphatic endothelial reprogramming of vascular endothelial cells by the Prox-1 homeobox transcription factor. EMBO J, 21(17): 4593-4599.

Rissanen TT, Markkanen JE, Gruchala M, Heikura T, Puranen A, Kettunen MI, et al. (2003). VEGF-D is the strongest angiogenic and lymphangiogenic effector among VEGFs delivered into skeletal muscle via adenoviruses. Circ Res, 92(10): 1098-1106.

Rubie C, Oliveira V, Kempf K, Wagner M, Tilton B, Rau B, et al. (2006). Involvement of chemokine receptor CCR6 in colorectal cancer metastasis. Tumour Biol, 27(3): 166-174.

Schoppmann SF, Birner P, Studer P, Breiteneder-Geleff S (2001). Lymphatic microvessel density and lymphovascular invasion assessed by anti-podoplanin immunostaining in human breast cancer. Anticancer Res, 21(4A): 2351-2355.

Shintani S, Li C, Ishikawa T, Mihara M, Nakashiro K, Hamakawa H (2004). Expression of vascular endothelial growth factor $\mathrm{A}, \mathrm{B}, \mathrm{C}$, and $\mathrm{D}$ in oral squamous cell carcinoma. Oral Oncol, 40(1): 13-20.

Sipos B, Kojima M, Tiemann K, Klapper W, Kruse ML, Kalthoff $\mathrm{H}$, et al. (2005). Lymphatic spread of ductal pancreatic adenocarcinoma is independent of lymphangiogenesis. J Pathol, 207(3): 301-312.

Straume O, Jackson DG, Akslen LA (2003). Independent prognostic impact of lymphatic vessel density and presence of low-grade lymphangiogenesis in cutaneous melanoma. Clin Cancer Res, 9(1): 250-256.

Sugiura T, Inoue Y, Matsuki R, Ishii K, Takahashi M, Abe $\mathrm{M}$, et al. (2009). VEGF-C and VEGF-D expression is 
Lymphangiogenesis, LECs and Lymphatic Metastasis in Head and Neck Cancer Z Zhang et al. http://www.ijos.org.cn

correlated with lymphatic vessel density and lymph node metastasis in oral squamous cell carcinoma: Implications for use as a prognostic marker. Int $J$ Oncol, 34(3): 673-680.

Thelen A, Jonas S, Benckert C., Weichert W, Schott E, Bötcher C, et al. (2009). Tumor-associated lymphangiogenesis correlates with prognosis after resection of human hepatocellular carcinoma. Ann Surg Oncol, 16(5): 1222-1230.

Trojan L, Michel MS, Rensch F, Jackson DG, Alken P, Grobholz R (2004). Lymph and blood vessel architecture in benign and malignant prostatic tissue: lack of lymphangiogenesis in prostate carcinoma assessed with novel lymphatic marker lymphatic vessel endothelial hyaluronan receptor (LYVE-1). $J$ Urol, 172(1): 103-107.

van der Auwera I, van Laere SJ, van den Eynden GG, Benoy I, van Dam P, Colpaert CG, et al. (2004). Increased angiogenesis and lymphangiogenesis in inflammatory versus noninflammatory breast cancer by real-time reverse transcriptase-PCR gene expression quantification. Clin Cancer Res, 10(23): 7965-7971.

Vlahakis NE, Young BA, Atakilit A, Sheppard D (2005). The lymphangiogenic vascular endothelial growth factors VEGF-C and -D are ligands for the integrin alpha9beta1. J Biol Chem, 280(6): 4544-4552.

Wen J, Fu AF, Chen LJ, Xie XJ, Yang GL, Chen XC, et al. (2009). Liposomal honokiol inhibits VEGF-D-induced lymphangiogenesis and metastasis in xenograft tumor model. Int J Cancer, 124(11): 2709-2718.

Williams CS, Leek RD, Robson AM, Banerji S, Prevo R, Harris AL, et al. (2003). Absence of lymphangiogenesis and intratumoural lymph vessels in human metastatic breast cancer. J Pathol, 200(2): 195-206.

Witmer AN, Vrensen GF, van Noorden CJ, Schlingemann
RO (2003). Vascular endothelial growth factors and angiogenesis in eye disease. Prog Retin Eye Res, 22(1): $1-29$.

Wong SY, Haack H, Crowley D, Barry M, Bronson RT, Hynes RO (2005). Tumor-secreted vascular endothelial growth factor-C is necessary for prostate cancer lymphangiogenesis, but lymphangiogenesis is unnecessary for lymph node metastasis. Cancer Res, 65(21): 97899798.

Xuan M, Fang YR, Wato M, Hata S, Tanaka A (2005). Immunohistochemical co-localization of lymphatics and blood vessels in oral squamous cell carcinomas. $J$ Oral Pathol Med, 34(6): 334-339.

Zhang B, Zhao WH, Zhou WY, Yu WS, Yu JM, Li S (2007). Expression of vascular endothelial growth factors-C and -D correlate with evidence of lymphangiogenesis and angiogenesis in pancreatic adenocarcinoma. Cancer Detect Prev, 31(6): 436-442.

Zhao D, Pan J, Li XQ, Wang XY, Tang C, Xuan M (2008). Intratumoral lymphangiogenesis in oral squamous cell carcinoma and its clinicopathological significance. $J$ Oral Pathol Med, 37(10): 616-625.

Zhu YM, Bagstaff SM, Woll PJ (2006). Production and upregulation of granulocyte chemotactic protein-2/ CXCL6 by IL-1beta and hypoxia in small cell lung cancer. Br J Cancer, 94(12): 1936-1941.

Zhuang Z, Jian P, Longjiang L, Bo H, Hongwei Z (2008). Identification of oral cancer cell-induced changes in gene expression profile of lymphatic endothelial cell. Cancer Invest, 26(10): 1002-1007.

Zhuang Z, Jian P, Longjiang L, Bo H, Wenlin X (2009). Altered phenotype of lymphatic endothelial cells induced by highly metastatic OTSCC cells contributed to the lymphatic metastasis of OTSCC cells. Cancer Sci, 2009 Nov 18. [Epub ahead of print].

*Corresponding authors: Long-jiang Li

Address: State Key Laboratory of Oral Diseases, West China Hospital of Stomatology, Sichuan University, No. 14, $3^{\text {rd }}$ Section, Renmin South Road, Chengdu 610041, China

Tel: $862885501440 \quad$ Fax: $862885582167 \quad$ E-mail: muzili63@163.com

International Journal of Oral Science has been accepted to be abstracted and indexed in CABI (Centre for Agriculture and Bioscience International)'s Quarterly Review.

- 14 - Int J Oral Sci, 2(1): 5-14, 2010 